\title{
Of hearts, minds, and guts: difficulties in diagnosing heart failure in the young
}

\section{Maria Frigerio ${ }^{1 *}$, Stefano Carugo $^{2}$, and Alessandra Voltolini ${ }^{3}$}

\author{
${ }^{1}$ 2nd Section of Cardiology, Heart Failure and Cardiac Transplant Unit, De Gasperis Cardio Center, Niguarda Hospital, Milan, Italy; ${ }^{2}$ Cardiology Unit, San Paolo Hospital, \\ Department of Health Sciences, University of Milan, Milan, Italy; and ${ }^{3}$ Clinical Psychology Service, Department of Mental Health, Niguarda Hospital, Milan, Italy
}

One year ago, a woman in her early twenties was diagnosed for the first time with dilated cardiomyopathy and low-output state at the emergency room of a city hospital, and was immediately transferred to the intensive care unit of a centre with advanced heart failure treatment and transplantation facilities. Due to refractory cardiogenic shock, she required temporary mechanical circulatory support and underwent emergency heart transplantation after 3 days. The explanted heart showed the typical pathological features of chronic dilated cardiomyopathy, with normal coronary arteries and no signs of recent/acute myocardial injury. Thus, what presented as a fulminant disease was indeed the terminal stage of a previously unrecognized chronic condition.

Delaying diagnosis of heart failure exposes patients to the risk of death, and precludes the opportunity for reverse remodelling and clinical improvement that can be observed with guideline-directed therapies in a non-negligible proportion of subjects, especially in women with non-ischaemic heart disease. ${ }^{1}$ Timely implementation of recommended management strategies relies largely upon the ability to identify the affected or possibly affected subjects in the primary care setting. We reconsidered the history of this patient, in order to identify factors that may reduce physicians' capability to diagnose heart failure, especially in the young.

\section{A matter of hearts and minds}

During the year preceding transplantation, the patient had complained several times with the family physician for shortness of breath on exertion, fatigue, difficulties in lying in bed and in getting asleep. The doctor attributed these symptoms to anxiety and dissatisfaction with patient's working and personal conditions. This woman was indeed labelled as a subject with a 'borderline personality disorder', after an episode of self-injury (self-inflicted superficial wounds) that had occurred when she was a teenager. Later on, upper abdominal pain, vomiting and weight loss (> $10 \mathrm{~kg}$ in 2 months) became the prominent symptoms. Patient's body weight had always been within the normal range, nevertheless there were eating behaviour irregularities. Thus, weight loss and hyporexia were also interpreted as due to psychological distress. Notably, at the time of self-injuries, the patient and her parents had shown fear and denial toward any psychiatric diagnosis, refusing psychological support and follow-up. Years later, they were profoundly frustrated by functional interpretation of patient's symptoms, and appeared somehow relieved when a physical diagnosis of heart disease had been finally made, despite its severity.

Non-suicidal self-injury is reported in more than $17 \%$ of adolescents, $13 \%$ of young adults, and $5 \%$ of adults, and at societal level there are strong implicit and explicit biases against these subjects. ${ }^{2}$ Patients with a history of psychiatric or behavioural disorders are known to be at risk for experiencing 'diagnostic overshadowing', meaning that the stigma of mental illness makes healthcare professionals to interpret reported physical symptoms as part of patients' mental health problems. As a consequence, they may be inadequately investigated and treated. ${ }^{3-5}$ Functional or mental interpretation of symptoms is more frequent in women than in men even in the absence of a psychiatric history, and gender bias is reported to influence medical decision in the field of ischaemic heart disease. ${ }^{6}$ Paradoxically, repeated, frequent reporting of the same symptoms to the family physician could have had the effect of making our patient to be considered as a 'complainer', thus receiving less rather than more attention over time.

\section{A matter of hearts and guts}

A few weeks before undergoing transplantation, our patient had accessed the emergency room of another hospital complaining for epigastric pain, and had been evaluated according to the 'chest pain pathway'. The electrocardiogram showed sinus tachycardia (120 b.p.m.), a prominent left atrial component of $P$ wave, and non-specific $\mathrm{T}$-wave abnormalities. The results of laboratory tests (including high-sensitivity troponin $\mathrm{T}$ but not natriuretic peptides) were normal. Cardiac ischaemia then had been ruled out, and the patient had been discharged with a diagnosis of 'functional

*Corresponding author. 2nd Section of Cardiology, Heart Failure and Cardiac Transplant Unit, De Gasperis Cardio Center, Niguarda Hospital, Piazza Ospedale Maggiore 3, 20162 Milan, Italy. Tel: +3902 64447791, Fax: +3902 64442566, Email: maria.frigerio@ospedaleniguarda.it 


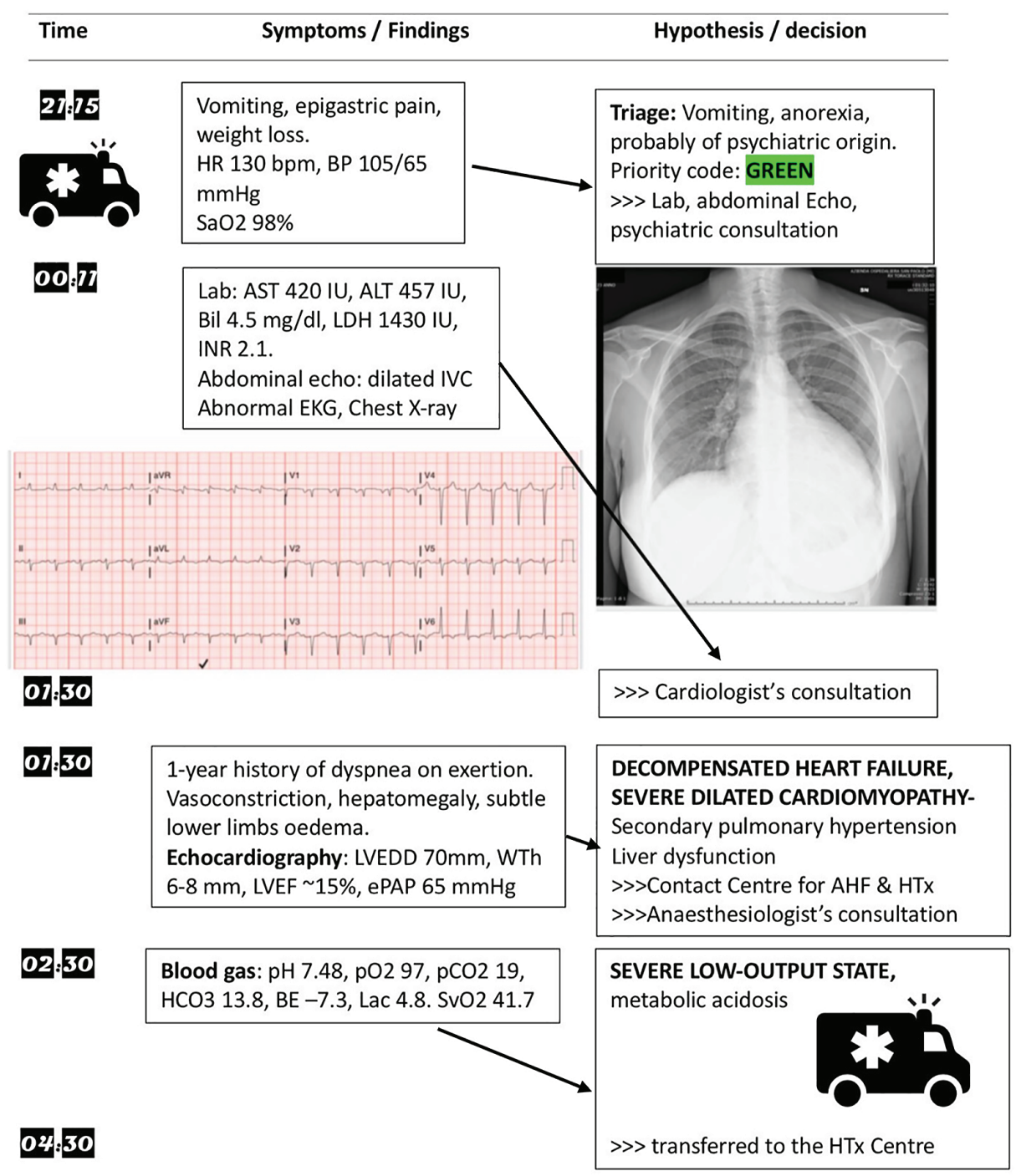

Figure 1 Summary of the process leading to diagnosis of heart failure in the emergency room. The figure shows how the diagnosis of severe, decompensated heart failure due to previously unrecognized dilated cardiomyopathy was reached after the patient accessed the emergency room complaining of epigastric pain, vomiting, and weight loss. The timeline is represented on the left, with black background. Main findings, their interpretation, and subsequent decisions are summarized in the boxes. Only most relevant pathological findings are reported. Green code assignment at triage means low short-term risk/low priority. Cardiomegaly and lung congestion were found at chest X-ray. Twelve-lead electrocardiogram (EKG) showed sinus tachycardia, left atrial enlargement, left axial deviation, non-specific ST-T abnormalities, suggesting left ventricular overload. Echocardiography recordings were not available. AHF, advanced heart failure; ALT, alanine amino transferase (normal values < $40 \mathrm{IU}$ ); AST, aspartate amino transferase (normal values < $40 \mathrm{IU}$ ); BE, base excess; Bil, bilirubin (normal values $<1 \mathrm{mg} / \mathrm{dL}$ ); $\mathrm{BP}$, blood pressure; echo, echotomography; ePAP, estimated pulmonary artery pressure; $\mathrm{HCO}_{3}$, bicarbonate; $\mathrm{HR}$, heart rate; $\mathrm{HTx}$, heart transplantation; INR, international normalized ratio; IVC, inferior vena cava; Lab, laboratory tests; Lac, lactate (normal values $\leq 2.5 \mathrm{mmoL} / \mathrm{L}$ ); LDH, lactate dehydrogenase (normal values < $400 \mathrm{IU}$ ); LVEDD, left ventricular end-diastolic diameter; LVEF, left ventricular ejection fraction; $\mathrm{pO}_{2}$, partial pressure of oxygen; $\mathrm{PCO}_{2}$, partial pressure of carbon dioxide; $\mathrm{SaO}_{2}$, arterial oxygen saturation; $\mathrm{SvO}_{2}$, mixed venous oxygen saturation.

dyspepsia'. Gastroenterological and psychiatric consultations had been suggested.

Feeding difficulties, loss of appetite, and delayed growth are typical of heart failure in the newborns, infants and children, ${ }^{7}$ while in adult patients dyspnoea and fatigue are the classic heart failure symptoms. ${ }^{1}$ It is not of common knowledge that teenagers and young adults may adapt over time to high left ventricular filling pressure, and present with symptoms and signs of visceral congestion rather than with dyspnoea, rales or wheezing, as pointed out in some studies. 8,9 Upper abdominal discomfort with epigastric pain that exacerbates after meals or on exercise, reduced food intake, and weight loss are due to liver and bowel congestion and/or to inadequate cardiac output, with an imbalance between requests and flow redistribution during digestion. Patients with 
these symptoms are often evaluated for gastrointestinal, liver, or biliary tract disease, but recommendations for work-up of epigastric or upper abdominal pain and dyspepsia do not list heart failure among their possible causes. ${ }^{10-12}$ On the other side, heart failure guidelines describe these symptoms mostly in the setting of chronic, advanced heart failure, and do not mention explicitly pain, which is relevant in patients' experience and, consequently, in their narration. ${ }^{1}$ Thus, currently available practice educational tools do not promote the awareness that upper abdominal symptoms and weight loss may be related to heart failure in newcomers, beyond the boundaries of paediatric age.

\section{A matter of doctors' minds}

In the night in which heart failure was finally diagnosed, at triage our patient had been classified with low priority, her symptoms being attributed to eating behaviour disturbances. Besides the mental illness bias, the absence of pulmonary rales and the lack of awareness of the possible presentation of heart failure with digestive symptoms among internists contributed to initial misinterpretation of the clinical picture, and to underestimation of its severity. Then tachycardia, electrocardiogram, liver dysfunction (which was absent at previous hospital access), and cardiomegaly at chest X-ray (that had not been performed at the other hospital) triggered cardiologist's consultation, and the right diagnosis was made (Figure 1). Notably, despite their good negative predictive value, ${ }^{1}$ natriuretic peptide levels were not assessed at both hospitals.

Missed or wrong diagnoses are probably underestimated, nevertheless they represent a relevant proportion of reported medical errors. ${ }^{13}$ Cognitive biases, gaps in knowledge and communication skills, time restraints, pitfalls in the flow of medical information, and other factors contribute to disguise physician's attention from the clues and links that lead to the right diagnosis. ${ }^{3,13}$ A common cause of misdiagnosis is the heuristic bias of going straightforwardly to the most probable condition and stick to it, disregarding alternative diagnosis. ${ }^{3}$ The growing amount of epidemiological data regarding various medical conditions contributes to our knowledge of clinical profiles and disease patterns, but, when approaching individuals, it must be remembered that they may or may not fit the average patient as characterized by cohort studies. In other words, physicians must ask themselves if symptoms and signs may suggest the presence of a serious - either frequent or infrequent - medical condition, a tip for medical reasoning called 'worst case scenario'. ${ }^{3}$ Everybody knows that heart failure is typically a disease of the elderlies, nonetheless we all must keep in mind also that it portends a high risk at any age: in several reports from randomized or observational studies, with an upper age limit of 40 to 50 years, only $1.5-2.5 \%$ of the patients were classified as 'young', and their 1-year mortality was between $6 \%$ and $12 \% .8,9,14$ Additionally, among patients discharged from the emergency room, the presence of dyspnoea or malaise/fatigue, i.e. of the commonest heart failure symptoms, was associated with a risk of dying within 1 week of more than three times the average, higher than that of patients with a definite diagnosis of heart failure ${ }^{15}$ - suggesting that heart failure is even more dangerous when unrecognized than when proven. Incidentally, the above quoted paper reported also that a diagnosis of mental illness was associated with a more than four-fold risk of dying early after discharge from the emergency room, ${ }^{15}$ indicating that these subjects require special attention - the opposite of what goes under the term of 'diagnostic overshadowing', 4,5

\section{Implications}

One year after transplantation, our patient is doing well, and is able to cope with her unusual condition, also with the help of regular psychological follow-up. In our opinion, stigma related to behavioural disturbances, lack of awareness of the possible relationship between abdominal symptoms and heart failure, inappropriate application of epidemiological knowledge when approaching individuals, plus perhaps gender bias, played a role in delaying the diagnosis of heart failure. The trajectory of this young woman suggests that more attention should be paid to qualitative and relational aspects of medical education and medicine practice. The awareness that in young adults heart failure may present with symptoms related to visceral congestion must be promoted among specialists and primary care physicians, and pertinent documents should be revised. Most of all, we should keep an open-minded, investigational attitude when approaching new patients, or new symptoms in patients we believe to know well, in order to discover important clues and understand their meaning, beyond what appears at first glance.

\section{Conflict of interest: none declared.}

\section{References}

1. Ponikowski P, Voors AA, Anker SD, Bueno H, Cleland JG, Coats AJ, Falk V, Gonzalez-Juanatey JR, Harjola VP, Jankowska EA, Jessup M, Linde C, Nihoyannopoulos P, Parissis JT, Pieske B, Riley JP, Rosano GM, Ruilope LM, Ruschitzka F, Rutten FH, van der Meer P. 2016 ESC guidelines for the diagnosis and treatment of acute and chronic heart failure: The Task Force for the diagnosis and treatment of acute and chronic heart failure of the European Society of Cardiology (ESC). Developed with the special contribution of the Heart Failure Association (HFA) of the ESC. Eur J Heart Fail 2016;18: 891-975.

2. Burke TA, Piccirillo ML, Moore-Berg SL, Alloy LB, Heimberg RG. The stigmatization of nonsuicidal self-injury. J Clin Psychol 2018;75:1-18.

3. Trowbridge RL. Twelve tips for teaching avoidance of diagnostic errors. Med Teach 2008:30:496-500.

4. Henderson C, Parke H, Clement S, Caffrey A, Gale-Grant O, Schulze B, Druss B, Thornicroft $\mathrm{G}$. Mental health-related stigma in health care and mental health-care settings. Lancet Psychiatry 2014;1:467-482.

5. Satcher D, Rachel SA. Promoting mental health equity: the role of integrated care. J Clin Psychol Med Settings 2017;24:182-186.

6. Biddle C, Fallavollita JA, Homish GC, Orom H. Gender bias in clinical decision making emerges when patients with coronary heart disease symptoms also have psychological symptoms. Heart Lung 2019;48:331-338.

7. Romer AJ, Rajagopal SK, Kameny RJ. Initial presentation and management of pediatric heart failure. Curr Opin Pediatr 2018;30:319-325.

8. Wong CM, Hawkins NM, Jhund PS, MacDonald MR, Solomon SD, Granger CB, Yusuf S, Pfeffer MA, Swedberg K, Petrie MC, McMurray J]. Clinical characteristics and outcomes of young and very young adults with heart failure. J Am Coll Cardiol 2013;62:1845-1854.

9. Wong CM, Hawkins NM, Ezekowitz JA, Jhund PS, Savu A, MacDonald MR, Kristensen SL, Petrie MC, McMurray J], McAlister FA, Kaul P. Heart failure in young adults is associated with high mortality: a contemporary population-level analysis. Can J Cardiol 2017;33:1472-1477.

10. Moayyedi PM, Lacy BE, Andrews CN, Enns RA, Howden CW, Vakil N. ACG and CAG clinical guideline: management of dyspepsia. Am J Gastroenterol 2017;112:988-1013. 
11. Tosetti C, Bellentani S, Benedetto E, Ubaldi E, Cardin F, Bozzani A; DyGeP Investigators and Researchers. The management of patients with new onset of upper gastro-intestinal symptoms in primary care. Dig Liver Dis 2010;42:860-864.

12. Robinson P, Perkins JC. Approach to patients with epigastric pain. Emerg Med Clin North Am 2016;34:191-210.

13. Royce CS, Hayes MM, Schwartzstein RM. Teaching critical thinking: a case for instruction in cognitive biases to reduce diagnostic errors and improve patient safety. Acad Med 2019;94:187-194.
14. Wong CM, Hawkins NM, Petrie MC, Jhund PS, Gardner RS, Ariti CA, Poppe KK, Earle N, Whalley GA, Squire IB, Doughty RN, McMurray J]; MAGGIC Investigators. Heart failure in younger patients: the Meta-analysis Global Group in Chronic Heart Failure (MAGGIC). Eur Heart J 2014;35: 2714-2721.

15. Obermeyer Z, Cohn B, Wilson M, Jena AB, Cutler DM. Early death after discharge from emergency departments: analysis of national US insurance claims data. BMJ 2017;356:j239. 